\title{
Reaction-rate theory with account of the crystal anharmonicity
}

\author{
V. I. Dubinko, ${ }^{1}$ P. A. Selyshchev, ${ }^{2}$ and J. F. R. Archilla ${ }^{3}$ \\ ${ }^{1}$ NSC Kharkov Institute of Physics and Technology, Akademicheskaya Str. 1, Kharkov 61108, Ukraine \\ ${ }^{2}$ Kiev National Taras Shevchenko University, Vladimirskaya Str. 64, Kiev 01033, Ukraine \\ ${ }^{3}$ Seville University, E-41011, Seville, Spain \\ (Received 19 June 2010; revised manuscript received 9 January 2011; published 25 April 2011)
}

\begin{abstract}
Reaction rate theory in solids is modified taking into account intrinsic localized modes or discrete breathers (DBs) that can appear in crystals with sufficient anharmonicity, resulting in violation of Arrhenius' law. Largeamplitude oscillations of atoms about their equilibrium positions in the lattice cause local potentials of alternating sign, which are described in terms of time-periodic modulations of the potential barriers for chemical reactions taking place in the vicinity of DBs. The reaction rate averaged over large macroscopic volumes and times including many DBs is increased by a factor that depends on the DB statistics. The breather statistics in thermal equilibrium and in thermal spikes in solids under irradiation with swift particles is considered, and the corresponding reaction rate amplification factors are derived.
\end{abstract}

DOI: 10.1103/PhysRevE.83.041124

PACS number(s): 05.40.-a, 63.20.Ry, 63.20.Pw

\section{INTRODUCTION}

The problem of escape from metastable states is of importance to many fields of physics, chemistry, engineering, and biology. It is well known that in thermal equilibrium the fluctuation-activated reaction rate $\dot{R}$ is expressed by Arrhenius' law:

$$
\dot{R}=R_{0} \exp \left(-E_{a} / k_{b} T\right),
$$

where $R_{0}$ and $E_{a}$ are the frequency factor and the activation energy, respectively, $k_{b}$ is the Boltzmann constant, and $T$ is the temperature. As shown below $R_{0}$ has dimensions of inverse time. How can the interplay of nonlinearity and discreteness of the lattice influence this law? It has been shown that in crystals with sufficient anharmonicity a special kind of time-periodic and spatially localized vibrations can appear, called intrinsic localized modes or discrete breathers (DBs) [1-5]. DBs have frequencies above or below the phonon band so that they do not couple with phonons and thus are thermally stable. Over the last decade much progress has been achieved in the understanding of DB properties and their role in various experimental situations. MacKay and Aubry [2] suggested that the existence of DBs could result in apparent violation of Arrhenius' law, that is, the phenomenon of chemical reactions taking place at much lower temperatures than expected. Further development of this hypothesis by Archilla et al. [3] has taken into account the DB statistics [4] for the evaluation of the reaction rate due to the DBs having energies above the activation energy. They have shown that, although there are many fewer breathers than phonons, there may be many more with energies above the activation energy, making them good candidates to explain, e.g., low-temperature reconstructive transformations observed in some layered insulators. In this paper we show that reaction rates depend on DBs of all energies due to effect of the time-periodic modulation of the activation energy. Large-amplitude oscillations of atoms about their equilibrium positions in the lattice cause local potentials of alternating sign, which may be described in terms of time-periodic modulations of the potential barriers for chemical reactions taking place in the vicinity of DBs.
The paper is organized as follows. In Sec. II we present a generic model for a Brownian particle escape from a potential well with a barrier height periodically modulated in time. In Sec. III we combine the reaction amplification rate due to one DB with the breather statistics in thermal equilibrium and in thermal spikes in solids under irradiation with swift particles. In Sec. IV we present a quantitative comparison of the proposed theory with experiments for equilibrium and nonequilibrium systems. In Sec. V we discuss possible extensions of the present model and some of the outstanding problems. We summarize in Sec. VI.

\section{ESCAPE RATE WITH ACCOUNT OF THE POTENTIAL BARRIER MODULATION}

Consider a heavily damped particle of mass $m$ and viscous friction $\gamma$, moving in a symmetric double-well potential $V(x)$ (see Fig. 1). The particle is subject to fluctuational forces that are, for example, induced by coupling to a heat bath. Such a model is archetypal for investigations in reaction-rate theory [6]. The fluctuational forces cause transitions between the neighboring potential wells with a rate given by the famous Kramers rate:

$$
\dot{R}_{K}=R_{0} \exp (-\Delta V / D), \quad R_{0}=\frac{\omega_{0} \omega_{b}}{2 \pi \gamma}
$$

with $\omega_{0}^{2}=V^{\prime \prime}\left(x_{m}\right) / m$ being the squared angular frequency of the potential in the potential minima, and $\omega_{b}^{2}=\left|V^{\prime \prime}\left(x_{b}\right) / m\right|$ the squared angular frequency at the top of the barrier; $\Delta V$ is the height of the potential barrier separating the two minima, and $D$ is the Gaussian white-noise strength, which is related to the temperature as $D=k_{b} T$ in the case of thermal equilibrium.

In the presence of periodic driving of frequency $\Omega$ and amplitude $V_{m}$, the double-well potential $V(x, t)=V(x)-$ $\left(x / x_{m}\right) V_{m} \cos (\Omega t)$ is tilted back and forth, thereby raising and lowering successively the potential barriers of the right and the left well, respectively, in an antisymmetric manner. If the driving frequency is small compared to the thermalization frequency, i.e., if $\Omega \ll \omega_{0}$, then periodically modulated escape 


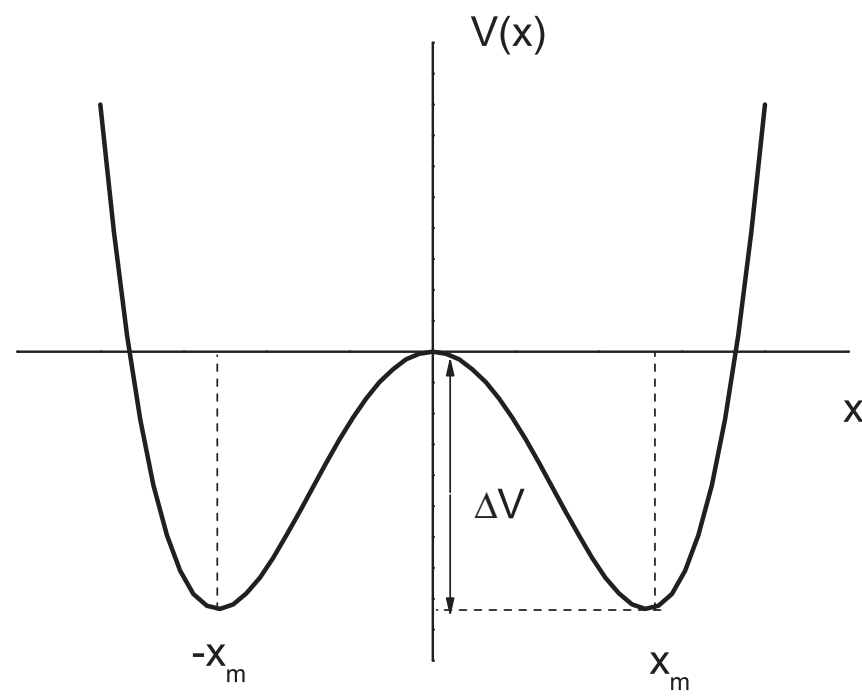

FIG. 1. Sketch of the double-well potential $V(x)=(1 / 4) b x^{4-}$ $(1 / 2) a x^{2}$. The minima are located at $\pm x_{m}$, where $x_{m}=(a / b)^{1 / 2}$. These are separated by a potential barrier with the height given by $\Delta V=$ $a^{2} / 4 b$.

rates of the Arrhenius type may be used (adiabatic assumption [7]):

$$
\dot{R}(t)=\dot{R}_{K} \exp \left[\frac{V_{m} \cos (\Omega t)}{D}\right] .
$$

This expression is widely used in the theory of stochastic resonance [7], which shows that noise-induced hopping between the potential wells can become synchronized with the weak periodic forcing. We are interested in another aspect of this problem, namely, in the rate of escape from the well averaged over the modulation period, $2 \pi / \Omega$, which is given by

$$
\begin{aligned}
\langle\dot{R}(t)\rangle & =\dot{R}_{K} \frac{\Omega}{2 \pi} \int_{0}^{2 \pi / \Omega} \exp \left[\frac{V_{m} \cos (\Omega t)}{D}\right] d t \\
& =\dot{R}_{K} I_{0}\left(\frac{V_{m}}{D}\right)
\end{aligned}
$$

where the amplification factor $I_{0}(x)$ is the zero-order modified Bessel function of the first kind.

In order to evaluate the average escape rate in a more general case of an arbitrary modulation frequency we will use another assumption, i.e., that the probability for a particle to escape from a well in each "jump" is given by $\exp (-\tilde{E})$, where $\tilde{E}$ is a random value that fluctuates around its mean value as $\tilde{E}=\langle E\rangle+E_{m} \cos (\tilde{\varphi})$ with a probability density for $\tilde{\varphi}$ given by $p(\tilde{\varphi})=1 / 2 \pi$. Then the integration over $\tilde{\varphi}$ will give for the average escape rate the same expression as in the adiabatic case:

$$
\begin{gathered}
\langle\dot{R}(\tilde{\varphi})\rangle=\dot{R}_{K} \frac{1}{2 \pi} \int_{0}^{2 \pi} \exp \left[E_{m} \cos (\tilde{\varphi})\right] d \tilde{\varphi}=\dot{R}_{K} I_{0}\left(E_{m}\right), \\
E_{m}=\frac{V_{m}}{D}
\end{gathered}
$$

This assumption is more general than the adiabatic one, since it requires essentially only the independence of the

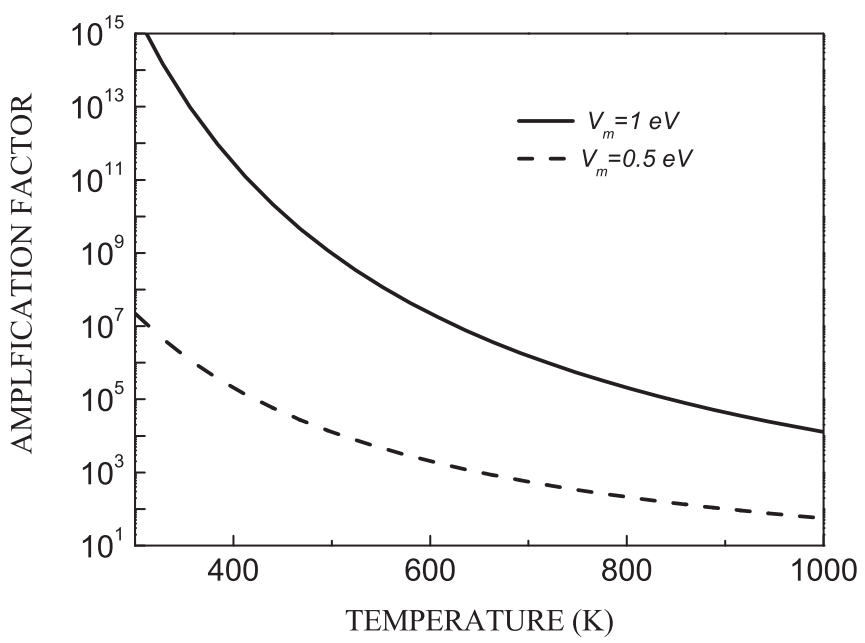

FIG. 2. Temperature dependence of the amplification factor $I_{0}$, for the average escape rate of a thermalized Brownian particle from a modulated potential barrier at different modulation amplitudes.

fluctuational force acting on a particle (characterized by the white noise) from the barrier modulation. In both cases the amplification factor is determined by the modulation to noise ratio, and it does not depend on the modulation frequency or the mean barrier height.

Thus, although the periodic forcing may be too weak to let the particle roll periodically from one potential well into the other one $\left(V_{m}<\Delta V\right)$, it can amplify the average reaction rate drastically if the ratio of the modulation amplitude to the noise strength is high enough, as is demonstrated in Fig. 2 for thermal equilibrium systems, in which $D=k_{b} T$.

\section{BREATHER-INDUCED AMPLIFICATION OF REACTION RATES}

Large-amplitude oscillations of atoms about their equilibrium positions in the lattice cause local potentials of alternating sign, which may be described in terms of timeperiodic modulations of the potential barriers for chemical reactions taking place in the vicinity of DBs. DBs may have frequencies above or below the phonon band depending on the system under consideration. Since the effective breather lifetime is much longer than the atomic oscillation period, we will assume the barrier modulation amplitude to be constant during the breather lifetime and to be proportional to the breather energy. Then the reaction rate at the breather sites will exceed that elsewhere by the amplification factor (4) determined by the breather energy $E_{B}$. We are interested in the reaction rate averaged over large macroscopic volumes and times including many DBs. Since DBs can appear at any lattice site randomly, this average can be found by multiplying the concentration (the mean number per site) of breathers of a given energy, $f_{B}(E)$, into the corresponding amplification factor, $I_{0}(E)$, and integrating 
over all possible DB energies normalized to the noise strength:

$$
\begin{aligned}
\langle\dot{R}\rangle_{B}= & \dot{R}_{K}\left[\int_{E_{\min }}^{E_{\mathrm{mod}}} f_{B}(E) I_{0}(E) d E\right. \\
& \left.+\int_{E_{\mathrm{mod}}}^{E_{\max }} f_{B}(E) I_{0}\left(E_{r}\right) d E\right], \quad E \equiv E_{B} / D, \\
& E_{\mathrm{mod}}=\left\{\begin{array}{l}
E_{\max }, \text { if } E_{\max } \leqslant E_{r}, \\
E_{r}, \text { if } E_{\max }>E_{r}
\end{array}\right.
\end{aligned}
$$

where $E_{r} \equiv \Delta V / D$ is the normalized reaction activation energy, and $E_{\text {mod }}$ is the maximum barrier modulation amplitude, which is assumed to be equal to the barrier height for breathers with energies higher than that.

In order to evaluate $f_{B}(E)$ we will use the DB statistics developed in Refs. [3,4] for two-dimensional breathers. This statistics theory is based on some simple hypotheses, which, in principle, can be fairly general: (1) DBs in two and three dimensions have a minimum energy $E_{\min }$, (2) the effective lifetime of a breather with energy $E$ is given by $\tau_{B}(E)=$ $\tau_{B}^{0}\left(\frac{E}{E_{\min }}-1\right)^{z}$, with $z$ and $\tau_{B}^{0}$ being constants (which means that they do not change with the energy $E$ ) that depend on the system. This law is the simplest mathematical expression that takes into account that large breathers have longer lives than smaller ones, with the lifetime of breathers with minimum energy $E_{\text {min }}$ equal to zero. It has to be considered as an approximation because it is not derived from first principles.

In these terms, the rate equation for $f_{B}(E, t)$ can be written as follows:

$$
\frac{\partial f_{B}(E, t)}{\partial t}=K_{B}(E)-\frac{f_{B}(E, t)}{\tau_{B}(E)},
$$

where $K_{B}(E)$ is the rate of creation of DBs with energy $E>E_{\min }$. It has an obvious steady-state solution at $\partial f_{B}(E, t) / \partial t=0$ :

$$
f_{B}(E)=K_{B}(E) \tau_{B}(E) .
$$

In the following sections we will consider the breather formation by thermal activation and then extend the model to nonequilibrium systems.

\section{A. DB formation by thermal activation}

The rate of creation of DBs is assumed to be proportional to $\exp \left(-E_{B} / k_{b} T\right)$ since breathers form from fluctuations through an activation process $[3,4]$. In normalized energy units one has

$$
K_{B}(E)=K_{B}^{0} \exp (-E), \quad E \equiv E_{B} / k_{b} T,
$$

whence it follows that under thermal equilibrium, the DB energy distribution function $f_{B}(E)$ and the mean number of breathers per site $n_{B}$ are given by

$$
\begin{gathered}
f_{B}(E)=K_{B}^{0} \tau_{B}^{0}\left(\frac{E}{E_{\min }}-1\right)^{z} \exp (-E), \\
n_{B}=\int_{E_{\min }}^{E_{\max }} f_{B}(E) d E \\
=K_{B}^{0} \tau_{B}^{0} \frac{\exp \left(-E_{\min }\right)}{\left(E_{\min }\right)^{z+1}} \int_{0}^{E_{\max }-E_{\min }} y^{z} \exp (-y) d y .
\end{gathered}
$$

Noting that $\Gamma(z+1, x)=\int_{0}^{x} y^{z} \exp (-y) d y$ is the second incomplete gamma function [3], Eq. (10) can be written as

$$
n_{B}=K_{B}^{0} \tau_{B}^{0} \frac{\exp \left(-E_{\min }\right)}{\left(E_{\min }\right)^{z+1}} \Gamma\left(z+1, E_{\max }-E_{\min }\right) .
$$

It can be seen that the mean DB energy is higher than the averaged energy density (or temperature):

$$
\left\langle E_{B}\right\rangle=k_{b} T\langle E\rangle=k_{b} T \frac{\int_{E_{\min }}^{E_{\max }} f_{B}(E) E d E}{\int_{E_{\min }}^{E_{\max }} f_{B}(E) d E} \rightarrow k_{b} T\left(E_{\min }+z+1\right) .
$$

So far we have followed the reasoning of Ref. [3], in which the mean reaction rate due to DBs is assumed to be determined by breathers with energies higher than the reaction activation energy (the potential barrier height), and hence it can be written as follows:

$$
\begin{aligned}
\langle\dot{R}\rangle_{B} & =R_{0}^{B} \int_{E_{r}}^{E_{\max }} f_{B}(E) d E \\
& =R_{0}^{B} K_{B}^{0} \tau_{B}^{0} \frac{\exp \left(-E_{\min }\right)}{\left(E_{\min }\right)^{z+1}} \int_{E_{r}-E_{\min }}^{E_{\max }-E_{\min }} y^{z} \exp (-y) d y,
\end{aligned}
$$

where $E_{r} \equiv \Delta V / k_{b} T$ is the normalized reaction activation energy and $R_{0}^{B}$ is the frequency factor that may be different from $R_{0}$ in Eq. (1) and it should be determined separately. It is evident that in this model, the breather effect on reaction rate vanishes if $E_{\max } \leqslant E_{r}$.

According to the present model, DBs of all energies influence the reaction rate due to their persistent character resulting in the reaction barrier modulation effect. Combining Eqs. (9) and (5), the mean reaction rate averaged over all breather energies takes the form

$$
\begin{aligned}
\langle\dot{R}\rangle_{B}= & \dot{R}_{K}\langle A\rangle_{B}, \quad E_{\bmod }=\left\{\begin{array}{l}
E_{\max }, \text { if } E_{\max } \leqslant E_{r} \\
E_{r}, \text { if } E_{\max }>E_{r}
\end{array}\right. \\
\langle A\rangle_{B}= & K_{B}^{0} \tau_{B}^{0}\left[\int_{E_{\min }}^{E_{\bmod }}\left(\frac{y}{E_{\min }}-1\right)^{z} \exp (-y) I_{0}(y) d y\right. \\
& \left.+\int_{E_{\bmod }}^{E_{\max }}\left(\frac{y}{E_{\min }}-1\right)^{z} \exp (-y) I_{0}\left(E_{r}\right) d y\right],
\end{aligned}
$$

where $\langle A\rangle_{B}$ is the averaged amplification factor.

In order to make quantitative estimates one has to know the product $K_{B}^{0} \tau_{B}^{0}$ and other parameters of DBs $\left(z, E_{\min }, E_{\max }\right)$ that depend on the system. We will use the results of numerical simulations [3], in which the numbers of breathers and their energy spectra have been calculated for a two-dimensional network of $50 \times 50$ nonlinear oscillators cooled down from the initial temperature of about $600 \mathrm{~K}$. The mean number of breathers per site $n_{B}$ was about $10^{-3}$, and according to the energy spectra, all types of multibreathers and single breathers with different symmetries have been formed with the following range of parameters: $z \approx 0.5-3, E_{\min } \approx 4-16$, $E_{\max } \approx 10-20$. In order to fit approximately the numerical form of $f_{B}(E)$, six breather types have been introduced, each one characterized by its own parameters $z, E_{\min }, E_{\max }$, and a relative probability to occur, $p$ (Table I). Substituting these data in Eq. (11), the product $K_{B}^{0} \tau_{B}^{0}$ can be estimated for each 
TABLE I. DB parameters deduced from numerical simulations [3] for six different DB types.

\begin{tabular}{lcccccc}
\hline \hline DB type & 1 & 2 & 3 & 4 & 5 & 6 \\
\hline$E_{B}^{\min }=E_{\min } k_{b} T(\mathrm{eV})$ & 0.24 & 0.37 & 0.41 & 0.62 & 0.67 & 0.83 \\
$z$ & 1.5 & 1.17 & 3 & 0.52 & 2.07 & 1.8 \\
$E_{B}^{\max }=E_{\max } k_{b} T(\mathrm{eV})$ & - & 0.47 & - & - & - & 0.95 \\
$p$ & 0.103 & 0.026 & 0.281 & 0.097 & 0.202 & 0.290 \\
$K_{B}^{0} \tau_{B}^{0}$ & 0.35 & 2.3 & 773 & $1.2 \times 10^{3}$ & $1.8 \times 10^{5}$ & $9 \times 10^{6}$ \\
\hline \hline
\end{tabular}

DB type and used for the evaluation of the DB amplification factor.

Figure 3 shows the amplification factor dependence on the reaction activation energy according to the present model and the model [3] assuming that all DBs have the same maximum energy. The comparison demonstrates that the modulation effect rapidly increases with increasing reaction barrier up to the maximum DB energy, above which it becomes the only mechanism of the reaction rate amplification. In this region the amplification factor does not depend on the activation energy.

In this section as in Refs. [3,4], the rate of breather creation was assumed to be proportional to $\exp \left(-E_{B} / k_{b} T\right)$. However, the necessary conditions for breather creation are not yet clear. Some studies suggest that DBs in thermal equilibrium will be generated only if the averaged energy density (or temperature) is large enough for nonlinear terms in the equations of motion to be relevant [8]. In the opposite case of rather small temperatures no breathers are expected to persist. As was argued in Ref. [4], the basic mechanism leading to energy localization is modulation instability of short-wavelength modes. The latter is more effective if dissipation of long-wavelength phonons is fast enough, which may explain the principal role of cooling (i.e., transient process of relaxation of a nonequilibrium state toward equilibrium) in the breather formation. A prominent example is the relaxation of an initially strongly heated lattice part modeled in Ref. [8]. In the following section we will consider a natural example of such processes that take place in solids under irradiation with swift particles. Apart from fundamental aspects, this example is of considerable technological importance in the fields of nuclear engineering and radiation effects.

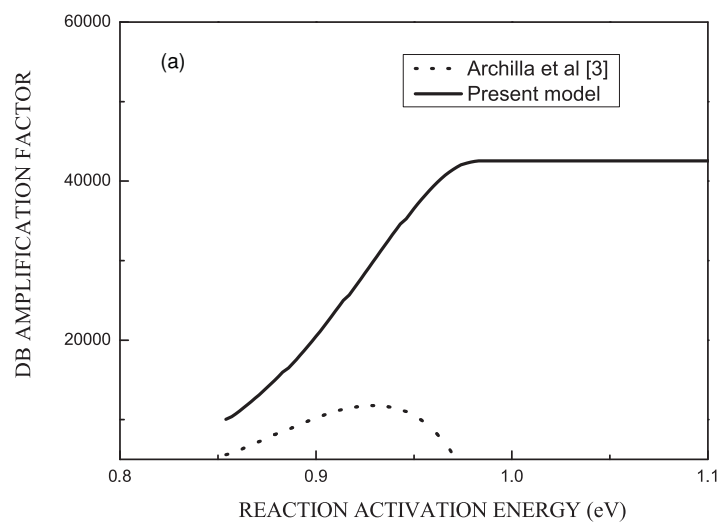

\section{B. DB formation in radiation-induced thermal spikes}

Penetrating into a solid, a heavy ion with the energy exceeding $1 \mathrm{MeV}$ /amu noticeably changes material properties in a narrow cylindrical region (track) around its trajectory. Since more than $90 \%$ of the energy loss of such projectiles is due to the electron excitations [9], these changes are often related to the local material heating called "thermal spike" (TS) resulting from energy and momentum transfer from the excited electrons to the lattice in the track [10,11]. As a result, thermally activated processes are enhanced, which should be taken into account in modeling of the radiation effects.

At the times of about $10^{-15} \mathrm{~s}$ electron gas within the track becomes thermalized, and its temperature can reach $10-100 \mathrm{eV}$ $[10,11]$. This thermal spike dissipates due to the heat diffusion in electron system and due to the heat transfer to the ion system, which is described conventionally by a set of two differential equations of the heat diffusion and exchange [12]:

$$
\begin{aligned}
& \frac{\partial T_{e}}{\partial t}=\chi_{e} \Delta T_{e}-\alpha_{e}\left(T_{e}-T_{p}\right) ; \quad \alpha_{e} \equiv \frac{\alpha_{e p}}{c_{e}}, \\
& \frac{\partial T_{p}}{\partial t}=\chi_{p} \Delta T_{p}+\alpha_{p}\left(T_{e}-T_{p}\right), \quad \alpha_{p} \equiv \frac{\alpha_{e p}}{c_{p}},
\end{aligned}
$$

subjected to the initial conditions $T_{e, p}(t=o)=\theta_{e, p}(\vec{r})$, where $c_{e, p}$ and $\chi_{e, p}$ are the heat capacity and conductivity of each subsystem, and $\alpha_{e p}$ is the electron-ion coupling coefficient.

Since the time of the energy transfer from a particle to the medium is much less that the relaxation times of the medium, one may consider the particle to be an instant source of energy that causes some initial temperature distribution in electron and phonon systems given by $\theta_{e, p}(\vec{r})$, where the subscript $e$

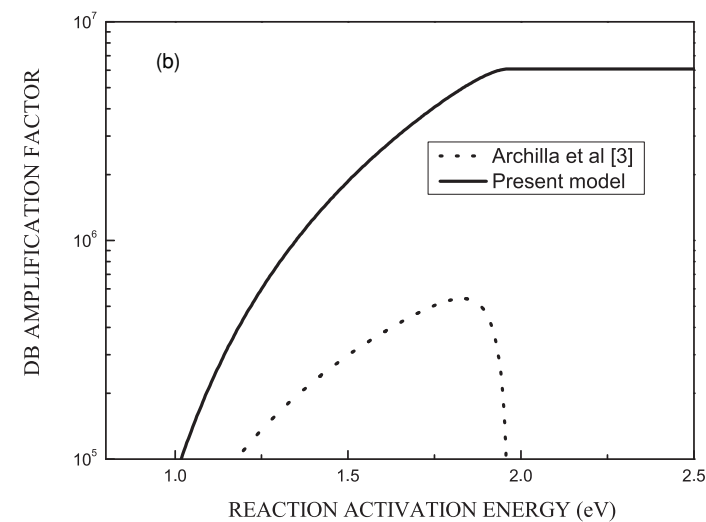

FIG. 3. Amplification factor dependence on the reaction activation energy at $T=600 \mathrm{~K}$ according to the present model [Eq. (15)] and the model [3] [Eq. (13)] assuming $R_{0}^{B}=R_{0}$ and $E_{B}^{\max }=1 \mathrm{eV}(\mathrm{a}) ; E_{B}^{\max }=2 \mathrm{eV}$ (b) for all breather types. Other DB parameters are presented in Table I. 
corresponds to electrons and $p$ corresponds to phonons. For a sufficiently long track in $Z$ direction with axial symmetry of the temperature distribution one has

$$
\theta_{e, p}(\vec{r})=\frac{\varepsilon_{e, p}}{c_{e, p} L} \frac{1}{4 \pi \rho_{0}^{2}} \exp \left(-\frac{\rho^{2}}{4 \rho_{0}^{2}}\right),
$$

where $\rho$ is the radius vector modulus, $\rho_{0}$ is the straggling coefficient, $L$ is the track length, and $\varepsilon_{e, p}$ is the energy transferred to electrons or phonons, respectively.

Assuming the coefficients $\alpha, \chi, c$ to be temperature independent, an analytical solution of the equation set could be obtained [12,13]. Consider two limiting cases, which allow making statistical analysis of the effect of thermal spikes on reaction rates that depend exponentially on the lattice temperature.

The limiting case, $\alpha_{e p} \rightarrow 0$, describes an adiabatic regime, in which there is no energy transfer between the subsystems, and the solution is given by [12]

$$
T_{e, p}(\rho, t)=\frac{\varepsilon_{e, p}}{4 \pi c_{e, p} L\left(\chi_{e, p} t+\rho_{0}^{2}\right)} \exp \left[-\frac{\rho^{2}}{4\left(\chi_{e, p} t+\rho_{0}^{2}\right)}\right] .
$$

This regime can be realized at short times at which the heat exchange between the subsystems can be neglected:

$$
t \ll t^{*}, \quad t^{*}=\left(\alpha_{e}+\alpha_{p}\right)^{-1},
$$

An opposite (asymptotic) regime, $\alpha_{e p} \rightarrow \infty$, can be realized at sufficiently large times and distances from the trek axis:

$$
t \gg t^{*}, \quad \rho \gg \rho^{*}, \quad\left(\rho^{*}\right)^{2}=\frac{\chi_{e}+\chi_{p}}{\alpha_{e}+\alpha_{p}} .
$$

In this regime, electron system cools down to the lattice temperature, and subsequently both systems have a common temperature, $T \approx T_{e} \approx T_{p}$, given by the following expression, which is similar to (19) but has effective heat conductivity, $\chi_{a} \equiv\left(\chi_{e} c_{e}+\chi_{p} c_{p}\right) / c_{a}$, and heat capacity, $c_{a} \equiv c_{e}+c_{p}$ :

$$
\begin{gathered}
T \approx \frac{1}{4 \pi L} \frac{\varepsilon_{a}}{c_{a}\left(\chi_{a} t+\rho_{0}^{2}\right)} \exp \left[-\frac{\rho^{2}}{4\left(\chi_{a} t+\rho_{0}^{2}\right)}\right], \\
\varepsilon_{a} \equiv \varepsilon_{e}+\varepsilon_{p} .
\end{gathered}
$$

Now, we are interested in the reaction rate, $\langle\dot{R}\rangle_{T}$, averaged over large macroscopic volumes and times $d V d t$ including many TSs. A mathematical scheme of this averaging procedure has been developed by Lifshits et al. [12]. It reduces the many-body problem of TSs in the phase volume $d V d t$ to the one-body problem, i.e., to the temperature field from one spike. The main point of this scheme is the determination of a four-dimensional volume in space and time, $\Omega(T)$, in which temperature is higher than $T$. In the case of cylindrical spike of a length $L, \Omega(T)$ is given by [12]

$$
\Omega(T)=\pi L \int_{0}^{t_{0}} \rho^{2}(T, t) d t,
$$

where $\rho(T, t)$ is the curve of constant temperature and $t_{0}(T)$ is the time at which $\rho(T, t)$ falls to zero. Then the mean reaction rate can be written in the following form:

$$
\begin{aligned}
&\langle\dot{R}(T)\rangle_{T}= \int_{0}^{\infty} \dot{R}(\bar{T}+T) P(T) d T, \quad P(T)=-\frac{d W(T)}{d T}, \\
& W(T) \equiv K_{T} \Omega(T)=\int_{T}^{\infty} P(\theta) d \theta
\end{aligned}
$$

where $W(T)$ is the probability of the temperature deviation from the mean value $\bar{T}$ by more than $T$ and $K_{T}=J / L$ is the TS production rate, which is proportional to the flux of energetic particles $\varphi$. The expression for $\langle\dot{R}(T)\rangle_{T}$ can be rewritten as the integral over $W$ :

$$
\langle\dot{R}(T)\rangle_{T}=\int_{0}^{1} \dot{R}[\bar{T}+T(W)] d W .
$$

Thus, in order to evaluate $\langle\dot{R}(T)\rangle_{T}$ one needs to derive the dependence $\rho(T, t)$ from the solution $T(\rho, t)$ given by Eqs. (19) and (22), and then solve the equation $\rho\left(T, t_{0}\right)=0$ with respect to $t_{0}$ and find expressions for $W(T)$ and $T(W)$. In the adiabatic regime this procedure results in the following expressions for $W\left(T_{e, p}\right)$ in each isolated subsystem [12]:

$$
\begin{gathered}
W\left(T_{e, p}\right) \approx\left(\frac{T_{e, p}^{*}}{T_{e, p}}\right)^{2}, \\
\left(T_{e, p}^{*}\right)^{2} \equiv \frac{1}{16 \pi}\left(\frac{\varepsilon_{e, p}}{L}\right)^{2} \frac{\varphi}{\chi_{e, p}\left(c_{e, p}\right)^{2}} .
\end{gathered}
$$

In the asymptotic regime one has a similar expression for a common temperature probability distribution [13]:

$$
W(T) \approx\left(\frac{T_{a}^{*}}{T}\right)^{2}, \quad\left(T_{a}^{*}\right)^{2} \equiv \frac{1}{16 \pi}\left(\frac{\varepsilon_{a}}{L}\right)^{2} \frac{\varphi}{\chi_{a} c_{a}^{2}} .
$$

Consider this regime in more details, since it describes the effect of thermal spikes in both subsystems on the reaction rate that depends on the lattice temperature. The substitution of (1) and (27) into (25) results in the following expression for the mean reaction rate:

$$
\langle\dot{R}(T)\rangle_{T} \approx[1-W(\bar{T})] \dot{R}(\bar{T})+2 R_{0}\left(\frac{k_{b} T_{a}^{*}}{E_{a}}\right)^{2} \Gamma\left(2, \frac{E_{a}}{k_{b} \bar{T}}\right),
$$

$$
\Gamma(2, x)=\int_{0}^{x} y \exp (-y) d y .
$$

The probability of the temperature deviation from the mean value is usually very small $[W(\bar{T})<<1]$, and so the first term in (28) corresponds simply to Arrhenius' law, which depends exponentially on the mean temperature. The second term in (28) describes the TS-induced addition to the mean reaction rate, $\langle\dot{R}(T)\rangle_{\mathrm{TS}}$. For sufficiently high activation energy $\left(E_{a} / k \bar{T} \gg 1\right)$ it takes an especially simple form:

$$
\begin{aligned}
\langle\dot{R}(T)\rangle_{T S} \approx 2 R_{0} & \left(\frac{k_{b} T_{a}^{*}}{E_{a}}\right)^{2} \Gamma\left(2, \frac{E_{a}}{k_{b} \bar{T}}\right) \underset{\frac{E_{a}}{k_{b} T} \gg 1}{\longrightarrow} \\
& 2 R_{0}\left(\frac{k_{b} T_{a}^{*}}{E_{a}}\right)^{2},
\end{aligned}
$$

which is proportional to the irradiation flux $J$ and does not depend on the lattice temperature. It is also proportional to the sum of the energies transferred to both subsystems squared, $\left(\varepsilon_{e}+\varepsilon_{p}\right)^{2}$. So even at $\varepsilon_{p}<<\varepsilon_{e}$, when the initial energy 


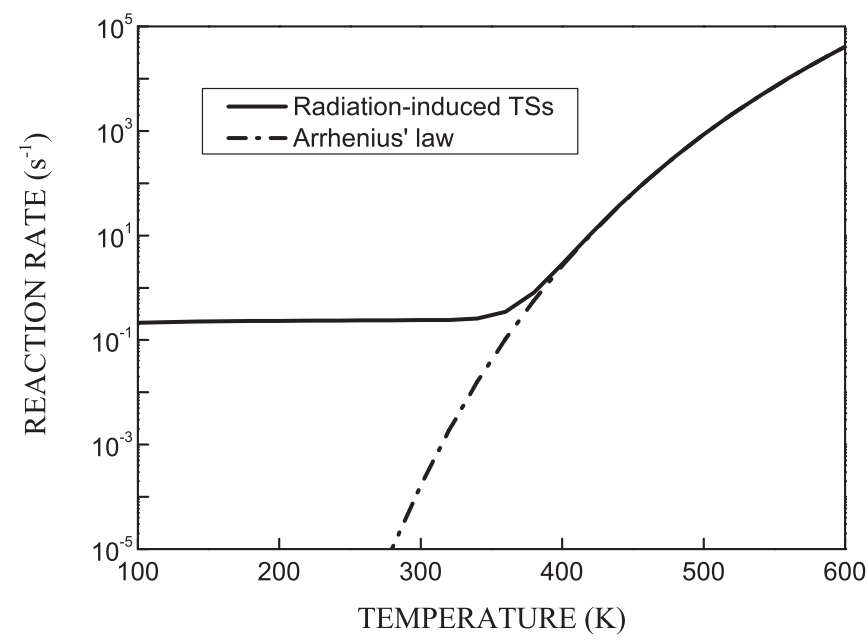

FIG. 4. Dependence of the mean reaction rate on the mean lattice temperature given by (28) for the case of ion irradiation of a metal target at the following irradiation and material parameters: $\varphi=10^{14}$ ion $/ \mathrm{cm}^{2} s, \varepsilon_{e}+\varepsilon_{p}=1 \mathrm{MeV}, L=2 \times 10^{-4} \mathrm{~cm}$, $A=10^{13} \mathrm{~s}^{-1}, E_{a}=1 \mathrm{eV}$.

transfer to the lattice is negligible, the reaction rate is increased due to the energy transfer via the electron subsystem. The TS effect on the reaction rate (1) is shown in Fig. 4 for the case of a typical ion irradiation.

It can be seen that the mean reaction rate becomes temperature independent below some threshold temperature $T_{\mathrm{TS}}$, which increases with increasing activation energy and irradiation flux as

$$
T_{\mathrm{TS}} \approx \frac{E_{a}}{k_{b} \ln \left[\frac{1}{2}\left(\frac{E_{a}}{k_{B} T_{a}^{*}}\right)^{2}\right]} .
$$

This fluctuating temperature background favors the DB creation in the cooling phase of radiation-induced thermal spikes. Averaging the local rate of creation of DBs (8) over large macroscopic volumes and times including many TSs one obtains the expression for the mean production rate of DBs with energy (normalized to the mean temperature) $E=$ $E_{B} / k_{b} \bar{T}$ :

$$
\left\langle K_{B}\right\rangle_{\mathrm{TS}}(E) \approx 2 K_{B}^{0}\left(\frac{T_{a}^{*}}{\bar{T}}\right)^{2} E^{-2} .
$$

Note that it is inversely proportional to the DB energy squared in contrast to the exponentially strong dependence of the local rate of creation of DBs. It follows from (31) [with account of (7)] that the energy distribution function and the mean steady-state concentration of breathers created in thermal spikes are given by

$$
\begin{gathered}
\left\langle f_{B}\right\rangle_{\mathrm{TS}}(E)=2 K_{B}^{0} \tau_{B}^{0}\left(\frac{T_{a}^{*}}{\bar{T}}\right)^{2}\left(\frac{E}{E_{\min }}-1\right)^{z} E^{-2}, \\
\left\langle n_{B}\right\rangle_{\mathrm{TS}}=2 K_{B}^{0} \tau_{B}^{0}\left(\frac{k_{b} T_{a}^{*}}{E_{B}^{\min }}\right)^{2} \int_{1}^{E_{\max } / E_{\min }} y^{-2}(y-1)^{z} d y .
\end{gathered}
$$

Temperature dependence of the mean concentration of DBs formed by thermal spikes (33) and by thermal fluctuations (9) is shown in Fig. 5. For the sake of simplicity, only one type of DB was selected with parameters that would correspond

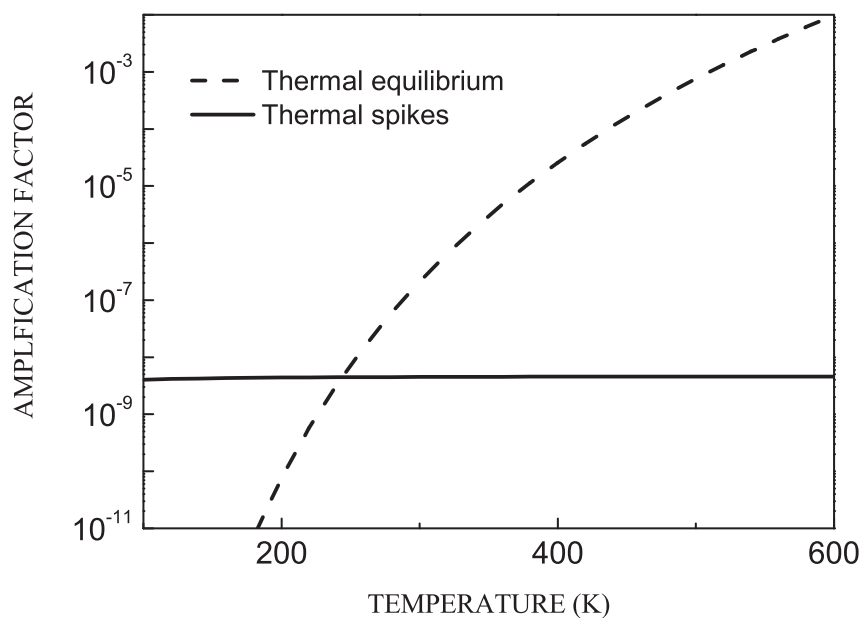

FIG. 5. Temperature dependence of the mean concentration of DBs formed by thermal fluctuations (9) and by thermal spikes (33) at the following irradiation and DB parameters: $\varphi=10^{14}$ ion $/ \mathrm{cm}^{2} \mathrm{~s}$, $\varepsilon_{e}+\varepsilon_{p}=1 \mathrm{MeV}, L=2 \times 10^{-4} \mathrm{~cm}, K_{B}^{0} \tau_{B}^{0}=1, \quad E_{B}^{\mathrm{min}}=0.2 \mathrm{eV}$, $E_{B}^{\max }=1 \mathrm{eV}, z=9$.

to the mean DB concentration of $\sim 10^{-3}$ with a mean DB energy of $\sim 0.7 \mathrm{eV}$ found in the numerical simulations [3] at $T \approx 550 \mathrm{~K}$.

Combining (32) and (5), the mean reaction rate averaged over all DBs created in TSs takes the form:

$$
\begin{aligned}
\langle\dot{R}(T)\rangle_{B, \mathrm{TS}}= & \dot{R}_{K} \int_{E_{\min }}^{E_{\max }}\left\langle f_{B}\right\rangle_{\mathrm{TS}}(E) I_{0}(E) d E=\langle A\rangle_{B, \mathrm{TS}} \dot{R}_{K}, \\
\langle A\rangle_{B, \mathrm{TS}}= & 2 K_{B}^{0} \tau_{B}^{0}\left(\frac{k_{b} T_{a}^{*}}{E_{B}^{\min }}\right)^{2} \\
& \times\left[\int_{1}^{E_{\bmod } / E_{\min }} y^{-2}(y-1)^{z} I_{0}\left(y E_{\min }\right) d y\right. \\
& \left.+\int_{E_{\bmod } / E_{\min }}^{E_{\max } / E_{\min }} y^{-2}(y-1)^{z} I_{0}\left(E_{r}\right) d y\right]
\end{aligned}
$$

Temperature dependence of the mean reaction rates with account of DBs formed by thermal fluctuations (14) and by thermal spikes (34) is shown in Fig. 6 along with the mean reaction rate due to TS without account of DBs (29). It can be seen that in spite of the fairly low concentration of DBs formed by thermal spikes, their contribution to the TS-induced enhancement of the reaction rates can be very significant.

Naturally, the DB effect depends on their parameters $\left(K_{B}^{0} \tau_{B}^{0}, z, E_{\min }, E_{\max }\right)$ that depend on the system. To show some of the trends let us express the Eq. (34) in the following form

$$
\begin{aligned}
& \langle\dot{R}(T)\rangle_{B, \mathrm{TS}}=\langle A\rangle_{B}\langle\dot{R}(T)\rangle_{\mathrm{TS}}, \\
\langle A\rangle_{B}= & K_{B}^{0} \tau_{B}^{0}\left(\frac{E_{a}}{E_{B}^{\min }}\right)^{2} \exp \left(-\frac{E_{a}}{k_{b} \bar{T}}\right) \\
& \times\left[\int_{1}^{E_{\mathrm{mod}} / E_{\min }} y^{-2}(y-1)^{z} I_{0}\left(y E_{\min }\right) d y\right. \\
& \left.+\int_{E_{\bmod } / E_{\min }}^{E_{\max } / E_{\min }} y^{-2}(y-1)^{z} I_{0}\left(E_{r}\right) d y\right],
\end{aligned}
$$



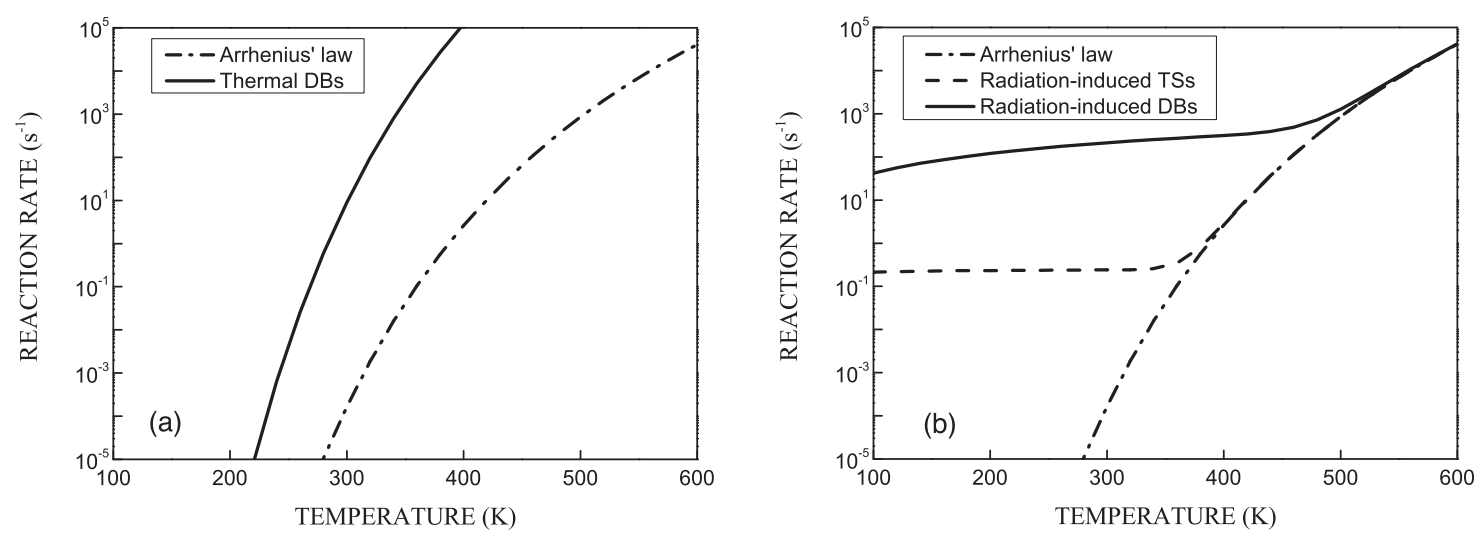

FIG. 6. Temperature dependence of the mean reaction rates with account of DBs formed at thermal equilibrium (a) and in thermal spikes (b) at the following irradiation and DB parameters: $\varphi=10^{14}$ ion $/ \mathrm{cm}^{2} \mathrm{~s}, \varepsilon_{e}+\varepsilon_{p}=1 \mathrm{MeV}, L=2 \times 10^{-4} \mathrm{~cm}, K_{B}^{0} \tau_{B}^{0}=1, E_{B}^{\mathrm{min}}=0.2 \mathrm{eV}$, $E_{B}^{\max }=E_{a}=1 \mathrm{eV}, z=9$.

where the amplification factor $\langle A\rangle_{B}$ does not depend on irradiation conditions and is determined only by DB parameters and by the reaction activation energy, similar to that for thermal equilibrium breathers defined by (15). Their comparison is shown in Fig. 7.

The common trend is a very strong dependence on the DB decay exponent, whereas the dependence on the maximum DB energy is qualitatively different. The concentration of high-energy breathers at thermal equilibrium (if any) is exponentially low, and so their contribution to the reaction rate is insignificant. On the other hand, the DB production rate in thermal spikes (31) is inversely proportional to the DB energy squared, and so high-energy breathers play a major role in the reaction rate amplification due to their relatively longer life times. Temperature dependence of the DB amplification factors is rather weak as demonstrated in Fig. 8 for two different values of the maximum DB energy.

\section{COMPARISON WITH EXPERIMENTAL DATA}

\section{A. Reconstructive transformations in mica muscovite}

Some silicates experience reconstructive transformations, which implies the breaking of the bond between silicon and oxygen, a particularly strong one. Therefore, high activation

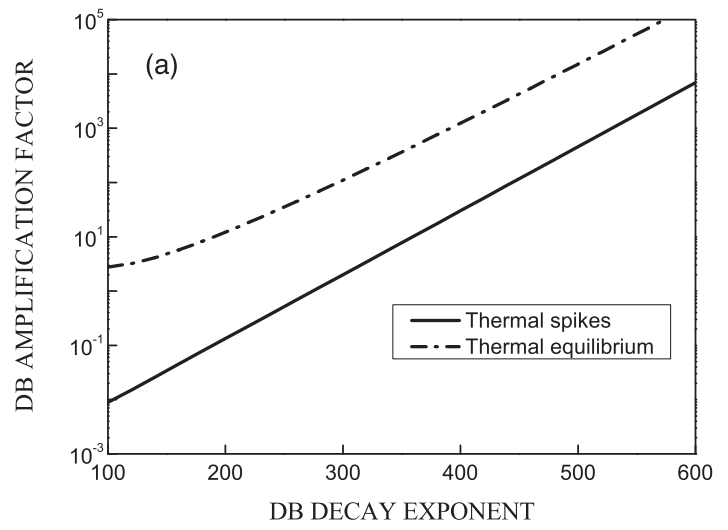

energies and a very slow reaction speed are expected. In the laboratory, temperatures above $1000{ }^{\circ} \mathrm{C}$ are necessary to observe the transformations in a relatively short period of time. In nature, many years are required. However, experiments in some layered silicates such as mica muscovite have been performed, in which about $36 \%$ of muscovite has been transformed into lutetium disilicate in three days at $300{ }^{\circ} \mathrm{C}$ [3]. The activation energy of the reconstructive transformations have been estimated to be $1-2 \mathrm{eV}$, which would require, according to Arrhenius' law (1), the transformation time larger than the experimental time by a factor of $10^{4}-10^{5}$. The lack of explanation from conventional chemical kinetics suggested the exploration of new hypotheses. In particular, the authors [3] explored the breather hypothesis assuming that in mica muscovite, there exist DBs that have enough energy to overcome the activation energy, and that there are enough of them to influence the reaction speed significantly. They concluded that the DB-induced increase of the reaction rate would be roughly equal to the ratio between the number of DBs and the number of phonons above the activation energy, and estimated the ratio to be about $10^{4}-10^{5}$, in agreement with experimental observations. However, the estimate was made assuming that the maximum DB energy was higher than the reaction barrier, which was not supported by numerical

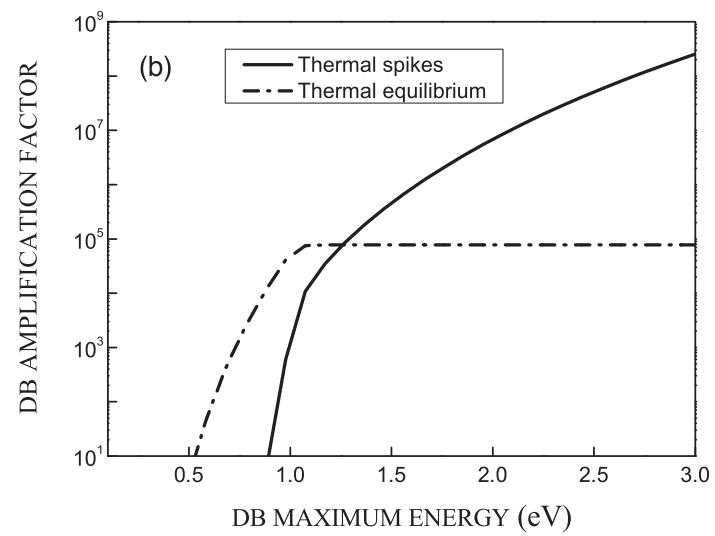

FIG. 7. Dependence of the DB amplification factors at thermal equilibrium (15) and under irradiation (37) at room temperature on the DB decay exponent and maximum energy at the following parameters: $K_{B}^{0} \tau_{B}^{0}=1, E_{B}^{\min }=0.2 \mathrm{eV}, E_{a}=1 \mathrm{eV}$; (a) $E_{B}^{\max }=E_{a}=1 \mathrm{eV}$; (b) $z=9$. 

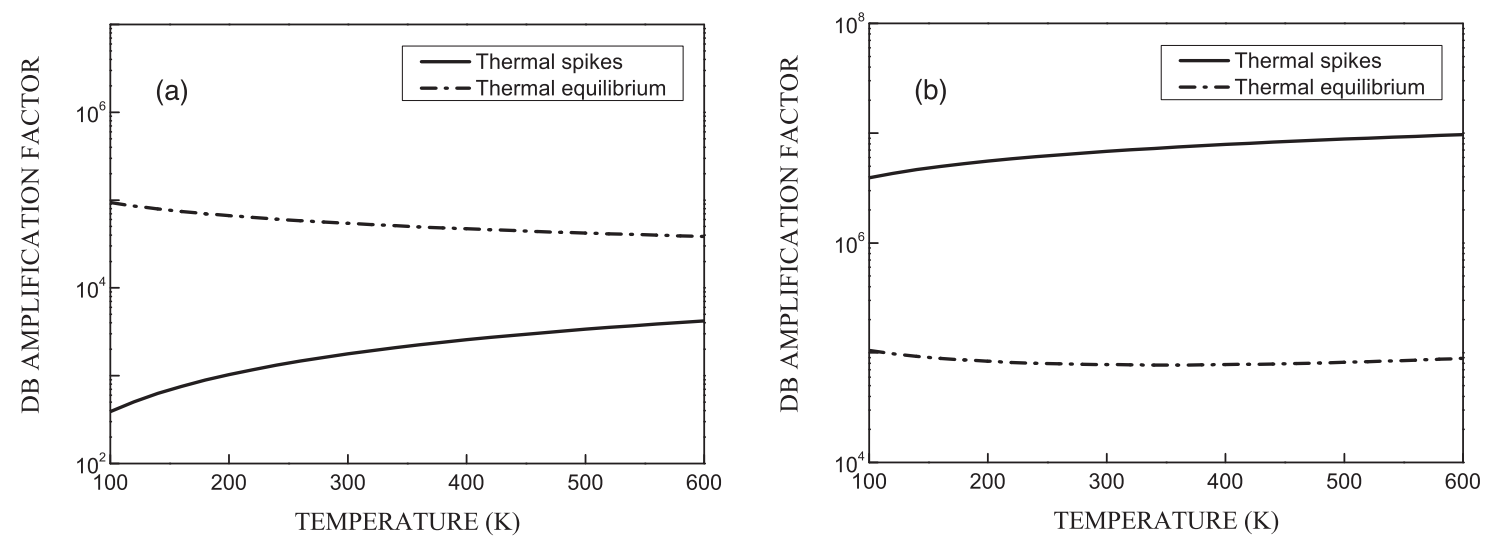

FIG. 8. Temperature dependence of the DB amplification factors at thermal equilibrium (15) and under irradiation (37) at the following parameters: $K_{B}^{0} \tau_{B}^{0}=1, E_{B}^{\min }=0.2 \mathrm{eV}, z=9, E_{a}=1 \mathrm{eV}$; (a) $E_{B}^{\max }=1 \mathrm{eV}$, (b) $E_{B}^{\max }=2 \mathrm{eV}$.

simulations (Table I), according to which the maximum DB energy was found to be about or below $1 \mathrm{eV}$ for some DB types and was not specified for others.

The present model seems to solve this problem by demonstrating that reaction rates are increased by DBs of all energies due to effect of the time-periodic modulation of the activation energy. Figure 3 shows that the DB amplification factor can be as high as $4 \times 10^{4}-6 \times 10^{6}$ even in the case of the activation energy being higher than the DB maximum energy, where the model [3] would give zero result. Thus we can conclude that the breather hypothesis can explain the low-temperature reconstructive transformations in layered insulators, which are good candidates for the DB formation under conditions close to thermal equilibrium. In the following section we consider the DB formation in strongly nonequilibrium systems, such as metals under irradiation with swift particles.

\section{B. Radiation-induced softening of metals}

Defects formed under irradiation in the bulk act as additional pinning centers for gliding dislocations, which results in the well-known effect of radiation-induced hardening [14]. On the other hand, there is experimental evidence of radiation-induced softening (RIS) under low-electron or gamma irradiation, which has been discovered in the early 1960s [15] and investigated extensively thereafter (see, e.g., Ref. [16]). Single HCP crystals of $\mathrm{Zn}, \mathrm{Sn}, \mathrm{In}$, and $\mathrm{Pb}$ have been irradiated at liquid nitrogen temperature $(78 \mathrm{~K})$ with electron flux density ranging from $10^{17} \mathrm{~m}^{-2} \mathrm{~s}^{-1}$ to $10^{18} \mathrm{~m}^{-2} \mathrm{~s}^{-1}$ and energies below and above the threshold displacement energies, the latter being $0.7 \mathrm{MeV}(\mathrm{Zn}), 0.8 \mathrm{MeV}$ (Sn, In) and $1.2 \mathrm{MeV}(\mathrm{Pb})$. At such low temperatures plastic strain occurs via dislocation glide, the rate of which is limited by thermally activated unpinning of dislocations from local obstacles. The over-threshold irradiation has resulted in the radiation-induced hardening due to formation of additional pinning centers. But with decreasing beam energy below the threshold level the plastic strain rate under irradiation increased as compared to that prior to or after irradiation. Then it was realized that irradiation not only produced defects, i.e., additional pinning centers for gliding dislocations, but also provided some softening mechanisms that operated during irradiation. However, the underlying RIS mechanism remained unclear.

Recent experiments by Dubinko et al. $[17,18]$ on the RIS of polycrystalline FCC metals $\mathrm{Al}$ and $\mathrm{Cu}$ (95.5\% pure) under electron irradiation were designed to test both subthreshold and over-threshold electron irradiation at ambient temperatures in order to make the results more closely related to the real in-reactor environment. The electron energy of $0.5 \mathrm{MeV}$ used in the experiments was higher than the threshold displacement energy in $\mathrm{Al}(0.15 \mathrm{MeV})$ and about the threshold displacement energy for $\mathrm{Cu}$. So in the first case, we dealt with the over-threshold electron irradiation, while in the second case, irradiation was essentially subthreshold. In spite of this difference, electron irradiation of $\mathrm{Al}$ and $\mathrm{Cu}$ has resulted in similar behavior of the deformation curves illustrated in Fig. 9 for the case of $\mathrm{Al}$.

The deformation strengthening curve $\sigma(\varepsilon)$ under continuous irradiation and a constant strain rate $\dot{\varepsilon}_{\text {ex }}$ goes below the control curve and up to the higher ultimate deformation before destruction [Fig. 9(a)], which means that the material under irradiation becomes more soft and less brittle. It should be noted that the softening occurs under over-threshold electron irradiation above the room temperature and the displacement rate, $K=2 \times 10^{-9} \mathrm{~s}^{-1}$, comparable to that in the nuclear reactor environment. The beam-induced temperature increase is about $20^{\circ} \mathrm{C}$ [Fig. 9(b)], which is insufficient to explain the observed material softening, but it makes it rather difficult to separate the heating effect from that of irradiation.

In order to single out an athermal component of the RIS, let us consider a discrete irradiation regime (Fig. 10), in which the samples were irradiated under external load during short time intervals followed by the intervals without irradiation.

When the electron beam is switched on $(\varphi \neq 0)$, the external stress jumps down sharply by the value $\delta \sigma_{\varphi}$, and subsequently a linear deformation stage occurs at which the deformation strengthening coefficient $\theta_{\varphi}$, is always lower than that without irradiation, and it becomes negative after some deformation level. This case is shown in detail in Fig. 10(b). When the electron beam is switched off $(\varphi=0)$, 


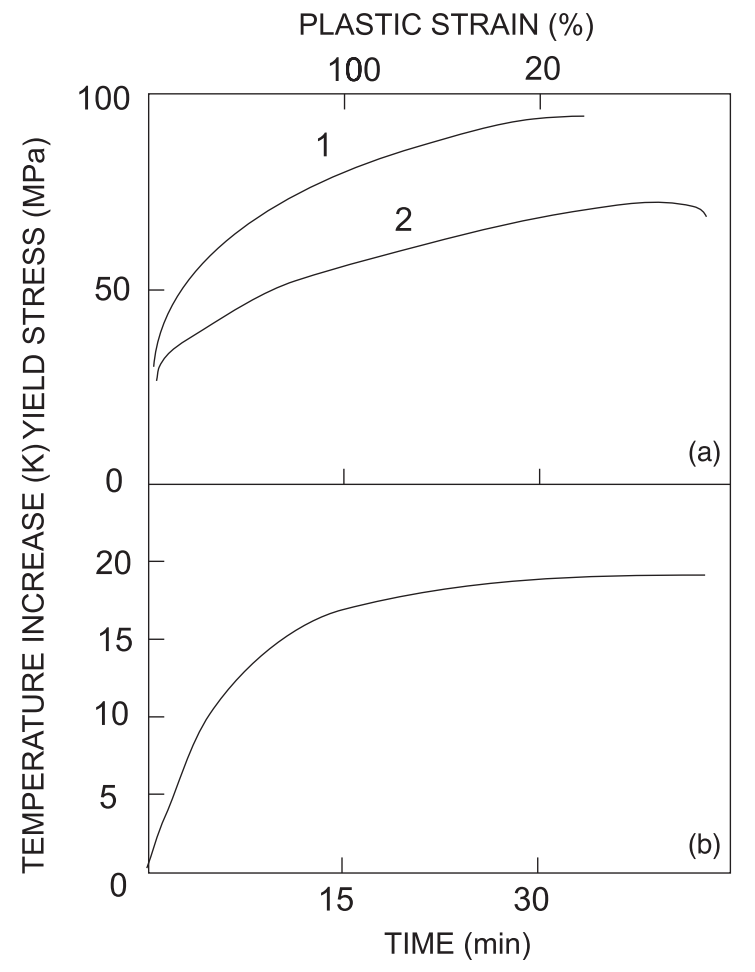

FIG. 9. The deformation strengthening curves, i.e., the dependence of the yield stress $\sigma$ on the plastic strain $\varepsilon$ and time for polycrystalline aluminum (99.5\%) [17]. The electron beam parameters are presented in Table II. (a) Curve 1 shows $\sigma(\varepsilon)$ without irradiation at room temperature and curve $2-\sigma(\varepsilon)$ under electron irradiation; (b) the time evolution of the sample temperature increases during irradiation.

the external stress jumps up sharply by the same value $\delta \sigma_{\varphi}$ and subsequently grows linearly with time at a rate $\theta_{0}>\theta_{\varphi}$. As a result, the net external stress decreases by the value $\Delta \sigma_{\varphi}$, which indicates that the metal microstructure changes due to irradiation.

The immediate stress jump at the moment of the beam switching on/off, $\delta \sigma_{\varphi}$, is a purely athermal effect since it is too quick for any significant temperature evolution. It increases with increasing deformation level, as shown in Fig. 11 for

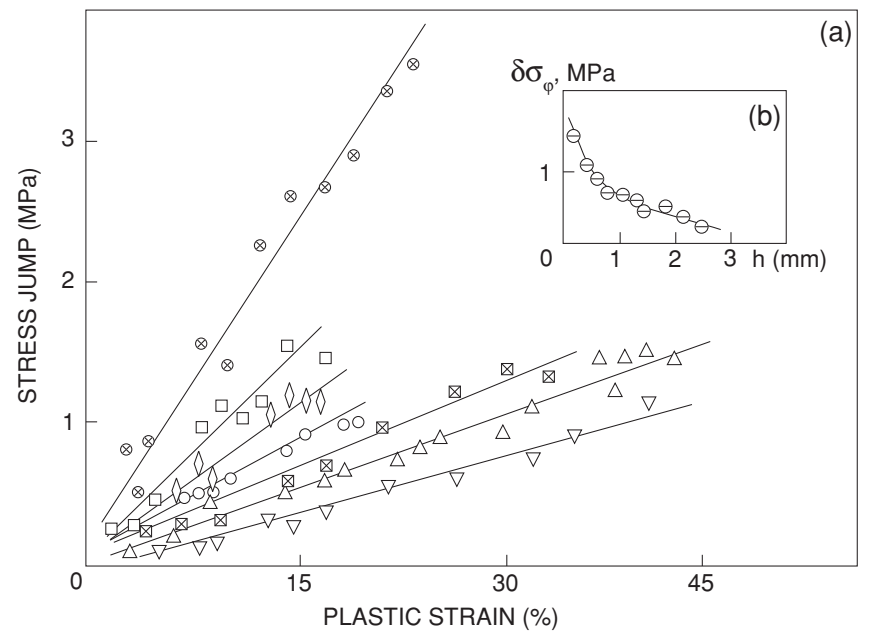

FIG. 11. (a) Stress jump $\delta \sigma_{\varphi}$ as a function of deformation of samples with different thickness $h: \mathrm{Cu}(\otimes, h=0.51 \mathrm{~mm}) ; \mathrm{Al}(\square, h$ $=0.23 ; \diamond, h=0.36 ; \bigcirc, h=0.57 ; \otimes, h=0.82 ; \triangle, h=1.76 ; \nabla, h$ $=2.15$ ). (b) $\delta \sigma_{\varphi}$ as a function of $h$ for $\mathrm{Al}$ at $\varepsilon=15 \%$.

$\mathrm{Cu}$ and $\mathrm{Al}$ samples of different thickness $h$. The electron penetration depth in $\mathrm{Al}$ is about $0.5 \mathrm{~mm}$, and $\delta \sigma_{\varphi}$ decreases by a factor of 8 with increasing sample thickness from 0.23 to $2.5 \mathrm{~mm}$ [Fig. 11(b)]. It can be seen that $\delta \sigma_{\varphi}$ increases linearly with increasing plastic strain, and it is higher in $\mathrm{Cu}$ than in $\mathrm{Al}: \delta \sigma_{\varphi}$ measured at $\varepsilon=15 \%$ is equal to $\sim 2.5$ $\mathrm{MPa}$ in $\mathrm{Cu}$ and $\sim 1 \mathrm{MPa}$ in $\mathrm{Al}$, which corresponds to approximately the same relative value of the stress jump, $\delta \sigma_{\varphi} / \sigma \sim 1 \%$.

Let us make an assumption that the electrons having energies in the $\mathrm{MeV}$ range produce thermal spikes, which help dislocations to overcome pinning centers resulting in the observed RIS. In order to evaluate this effect we will use a classical "string" model of the dislocation employed recently for modeling of the plasticity increase of materials under electric current pulses [19]. Accordingly, the rate of
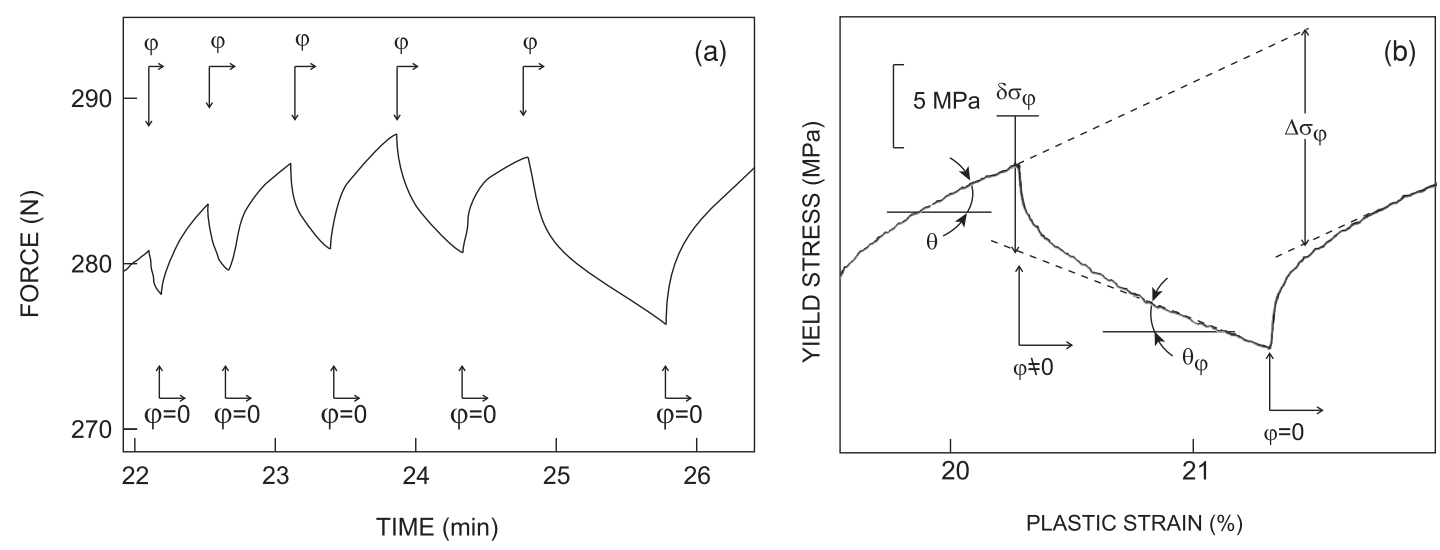

FIG. 10. (a) Evolution of the external tensile force (measured in newtons) during deformation of Al samples under discrete irradiation regime; (b) yield stress evolution during one irradiation period at a late deformation stage. Intervals designated with $\varphi=0$ correspond to zero electron flux. 
thermally activated plastic strain is given by the well-known expression

$$
\begin{gathered}
\dot{\varepsilon}_{T}=\dot{\varepsilon}_{0} \exp \left[-\frac{E_{a}(\sigma)}{k_{b} T}\right], \quad \dot{\varepsilon}_{0}=b l_{d} \rho_{d} \omega_{d}, \\
\omega_{d} \approx \frac{2}{\pi b x_{\mathrm{cr}}} \sqrt{\frac{H_{0} \omega_{D} b}{\rho v_{s}}},
\end{gathered}
$$

where $b$ is the Burgers vector length, $\rho_{d}$ is the dislocation density, $l_{d}$ is the mean length of dislocation segments pinned to the obstacles, $\omega_{D}$ and $\omega_{d}$ are the Debye frequency and the frequency of the dislocation oscillations, respectively, $\rho$ is the material density, $v_{s}$ is the sound velocity, and $x_{\mathrm{cr}}$ and $E_{a}(\sigma)$ are, respectively, the critical distance and effective activation energy required to unpin the dislocation from an obstacle, which decreases with increasing external stress $\sigma$ as follows:

$$
\begin{gathered}
E_{a}(\sigma)=H_{0}\left(1-\frac{\sigma_{\text {eff }}}{\sigma_{c}}\right)^{2}, \quad \sigma_{\text {eff }}=\sigma-\sigma_{\text {in }}, \\
\sigma_{c}=\kappa \sqrt{c_{I}}, \quad \sigma_{\text {in }}=\frac{\mu b}{2 \pi} \sqrt{\rho_{d}},
\end{gathered}
$$

where $H_{0}$ is the activation energy required to unpin the dislocation from an obstacle at zero effective stress, $\sigma_{\text {eff }}$, and $\sigma_{c}$ is the critical stress to unpin the dislocation at zero temperature (neglecting quantum oscillations), $\sigma_{\text {in }}$ is the internal stress due to the dislocation microstructure, $\mu$ is the shear modulus, $\kappa$ is the phenomenological coefficient proportional to the shear modulus [20]. The mean length of dislocation segments $l_{d}$ is determined by the dislocation density and the concentration of impurity atoms $c_{I}$ acting as pinning centers:

$$
l_{d}\left(\rho_{d}, c_{I}\right)=\left(\sqrt{\rho_{d}}+\frac{1}{b} \sqrt{c_{I}}\right)^{-1} .
$$

Now, in order to evaluate the yield stress under a constant strain rate one has to solve Eq. (38) with respect to $\sigma$, which results in the well-known expression describing the deformation strengthening dependence on temperature and the strain rate $\dot{\varepsilon}_{\text {ex }}$ :

$$
\sigma_{T}\left(T, \dot{\varepsilon}_{\mathrm{ex}}\right)=\sigma_{\mathrm{in}}+\sigma_{c}\left[1-\sqrt{\frac{k_{b} T}{H_{0}} \ln \left(\frac{\dot{\varepsilon}_{0}}{\dot{\varepsilon}_{\mathrm{ex}}}\right)}\right] .
$$

With account of the irradiation-induced TSs, substituting (38) for (1), one can rewrite the expression (29) as

$$
\langle\dot{\varepsilon}\rangle_{\mathrm{TS}} \approx 2 \dot{\varepsilon}_{0}\left[\frac{k_{b} T_{a}^{*}}{E_{a}(\sigma)}\right]^{2}, \quad T_{a}^{*}(\varphi)=\sqrt{\frac{1}{16 \pi}\left(\frac{\varepsilon_{a}}{L}\right)^{2} \frac{\varphi}{\chi_{a} c_{a}^{2}}},
$$

whence the TS-induced yield stress can be derived:

$$
\sigma_{\mathrm{TS}}\left(\varphi, \dot{\varepsilon}_{\mathrm{ex}}\right)=\sigma_{\mathrm{in}}+\sigma_{c}\left[1-\left(\frac{2 \dot{\varepsilon}_{0}}{\dot{\varepsilon}_{\mathrm{ex}}}\right)^{1 / 4} \sqrt{\frac{k_{b} T_{a}^{*}(\varphi)}{H_{0}}}\right],
$$

which, in contrast to (41), depends on the irradiation flux $\varphi$.

The plastic strain rate with account of the TS-induced DBs is given by the expression similar to (36):

$$
\langle\dot{\varepsilon}\rangle_{B, \mathrm{TS}}=\langle A\rangle_{B}\langle\dot{\varepsilon}\rangle_{\mathrm{TS}},
$$

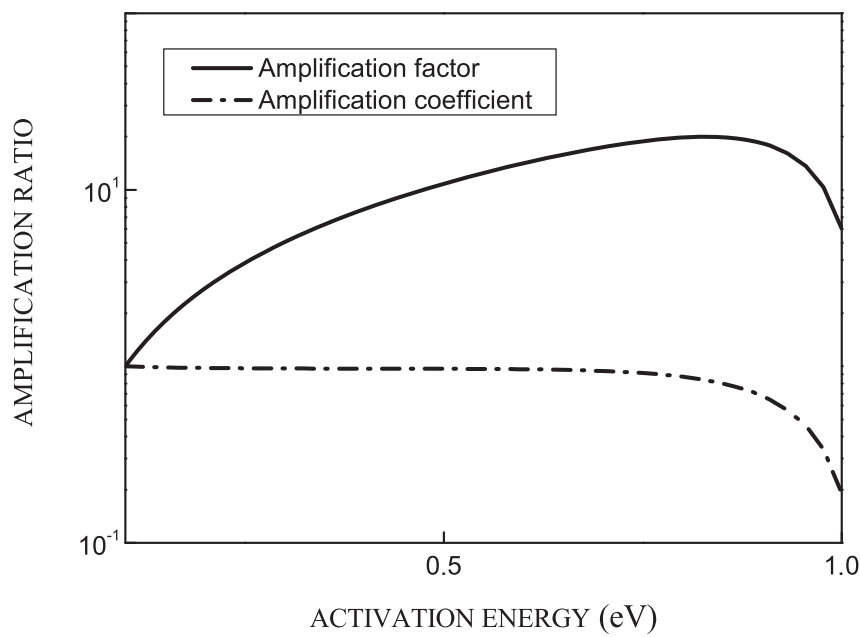

FIG. 12. Dependence of the relative DB amplification factor, $\langle A\rangle_{B}(E) /\langle A\rangle_{B}\left(E_{\min }\right)$, on the reaction activation energy $E$ at the following DB parameters: $\mathrm{z}=9, E_{B}^{\min }=0.1 \mathrm{eV}, E_{B}^{\max }=1 \mathrm{eV}$. Dotted curve corresponds to the relative DB amplification coefficient, $A_{B}^{0}(E) / A_{B}^{0}\left(E_{\min }\right)$.

where the amplification factor, $\langle A\rangle_{B}$, does not depend on irradiation conditions, but it depends on the reaction activation energy, $E_{a}(\sigma)$, in a complicated way. However, as demonstrated in Fig. 12, this dependence can be approximated as

$$
\langle A\rangle_{B} \approx A_{B}^{0} E_{a}^{3 / 2}, \quad E_{a}(\sigma)<E_{B}^{\max }
$$

for activation energies small as compared to the DB maximum energy, whence the DB-induced yield stress can be evaluated by the expression

$$
\sigma_{\mathrm{DB}}\left(\varphi, \dot{\varepsilon}_{\mathrm{ex}}\right)=\sigma_{\mathrm{in}}+\sigma_{c}\left\{1-A_{B}^{0}\left(\frac{2 \dot{\varepsilon}_{0}}{\dot{\varepsilon}_{\mathrm{ex}}}\right) \frac{\left[k_{b} T_{a}^{*}(\varphi)\right]^{2}}{\sqrt{H_{0}}}\right\},
$$

where $A_{B}^{0}$ is the constant amplification coefficient determined only by the DB parameters presented in Table II. The above evaluated yield stresses are valid for single crystals, whereas for polycrystalline materials they should be multiplied by the Taylor factor $M(M=3.06$ for equiaxed bcc and fcc metals [14]).

The immediate yield stress jump at the moment of the beam switching on/off occurs so quickly that the sample temperature and internal stress (determined by the microstructure) remains constant. Its magnitude, $\left|\delta \sigma_{\varphi}\right|$, depending on the underlying mechanism, is given by the difference between the expressions (41), (43), and (46) for zero and nonzero irradiation flux $\varphi$. Accordingly, the classical expression (41), which does not depend on $\varphi$, would predict no RIS effect: $\left|\delta \sigma_{\varphi}\right|_{T}=0$, while the other two expressions result in the following RIS effects for polycrystalline materials:

$$
\begin{gathered}
\left|\delta \sigma_{\varphi}\right|_{\mathrm{TS}}\left(\varphi, \dot{\varepsilon}_{\mathrm{ex}}\right)=M \sigma_{c}\left(\frac{2 \dot{\varepsilon}_{0}}{\dot{\varepsilon}_{\mathrm{ex}}}\right)^{1 / 4} \sqrt{\frac{k_{b} T_{a}^{*}(\varphi)}{H_{0}}} \propto \rho_{d}^{1 / 4} \varphi^{1 / 4}, \\
\left|\delta \sigma_{\varphi}\right|_{\mathrm{DB}}\left(\varphi, \dot{\varepsilon}_{\mathrm{ex}}\right)=M \sigma_{c} A_{B}^{0}\left(\frac{2 \dot{\varepsilon}_{0}}{\dot{\varepsilon}_{\mathrm{ex}}}\right) \frac{\left[k_{b} T_{a}^{*}(\varphi)\right]^{2}}{\sqrt{H_{0}}} \propto \rho_{d} \varphi .
\end{gathered}
$$


TABLE II. Material and irradiation parameters used in calculations.

\begin{tabular}{ll}
\hline \hline Parameter & Value \\
\hline Atomic spacing, $b(\mathrm{~m})$ & $3.23 \times 10^{-10}$ \\
Atomic volume of the host lattice, $\omega\left(\mathrm{m}^{-3}\right)$ & $2.36 \times 10^{-29}$ \\
Matrix shear modulus, $\mu(\mathrm{GPa})$ & $26.5(\mathrm{Al}) ; 54.6(\mathrm{Cu})$ \\
Critical stress coefficient, $\kappa(\mathrm{MPa})$ & $110(\mathrm{Al}) ; 248(\mathrm{Cu})$ \\
Activation energy, $H_{0}=\mu \omega / 4$ & $0.977(\mathrm{Al}) ; 2.012(\mathrm{Cu})$ \\
Critical unpinning distance, $x_{\mathrm{cr}}$ & $x_{\mathrm{cr}}=4 b$ \\
Concentration of impurity pinning centers, $c_{I}$ & $5 \times 10^{-3}$ \\
Initial dislocation density, $\rho_{d}^{0}\left(\mathrm{~m}^{-2}\right)$ & $10^{12}$ \\
Mean dislocation propagation range, $L_{\mathrm{eff}}(\mu \mathrm{m})$ & 2.6 \\
Debye temperature, $T_{D}(\mathrm{~K})$ & $394(\mathrm{Al}) ; 315(\mathrm{Cu})$ \\
Minimum DB energy, $E_{B}^{\min }=\mu \omega / 44(\mathrm{eV})$ & $0.089(\mathrm{Al}) ; 0.183(\mathrm{Cu})$ \\
Maximum DB energy, $E_{B}^{\min }=\mu \omega / 6(\mathrm{eV})$ & $0.651(\mathrm{Al}) ; 1.342(\mathrm{Cu})$ \\
DB decay exponent, $\mathrm{z}$ & 9 \\
Electron beam energy, $\varepsilon_{a}(\mathrm{MeV})$ & 0.5 \\
Electron beam density, at $\varphi\left(\mathrm{m}^{-2} \mathrm{~s}^{-1}\right)$ & $4 \times 10^{17}$ \\
Ambient temperature, $T(\mathrm{~K})$ & 300 \\
Plastic strain rate, $\dot{\varepsilon}_{\mathrm{ex}}$ & $10^{-4}$ \\
\hline
\end{tabular}

As can be seen, the TS-induced RIS effect depends on the irradiation and loading conditions rather weakly, as compared to the linear dependence of the DB-induced RIS effect on the irradiation flux and dislocation density. The latter is proportional to the plastic strain $\varepsilon$, according to the classical Taylor model [21]:

$$
\varepsilon=b \rho_{d} L_{\mathrm{eff}}
$$

where $L_{\text {eff }}$ is the mean propagation distance of gliding dislocations before they get stuck in the dislocation "forest," which is typically in the micron range (Table II). Accordingly, the RIS effect is expected to increase with increasing plastic strain, in agreement with experimental data (Fig. 11). A more detailed comparison between the theory and experiment [Fig. 13(a)] shows that the TS-induced RIS mechanism operates only at

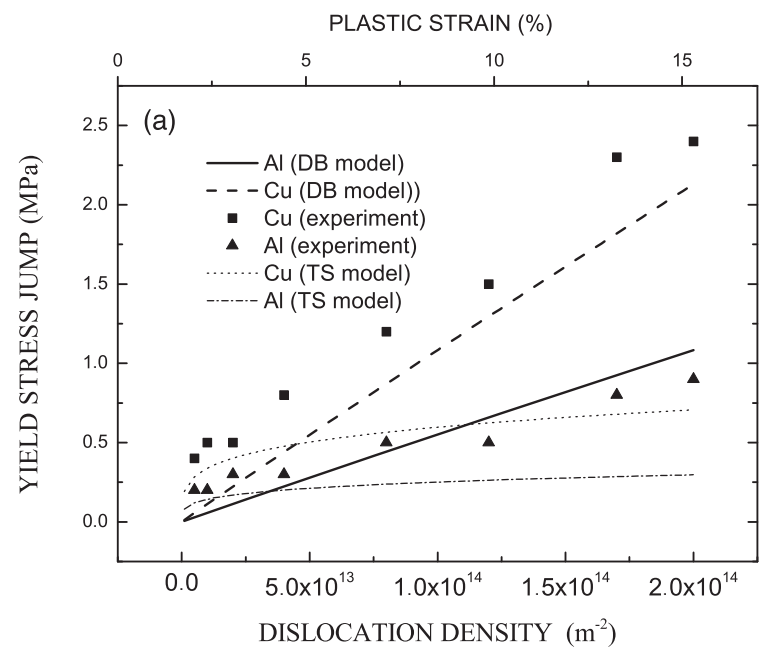

low strain levels, while the DB-induced RIS mechanism is responsible for the observed liner dependence of the RIS on the plastic strain. Figure 13(b) demonstrates that both mechanisms are temperature independent in agreement with their athermal nature.

These results show that mechanical properties of materials under reactor conditions can be different from those of the surveillance samples in out-reactor tests after an equivalent irradiation dose, which should be taken into account in forecasting the material service life.

\section{DISCUSSION}

In this section we will discuss some perspective extensions of the present model.

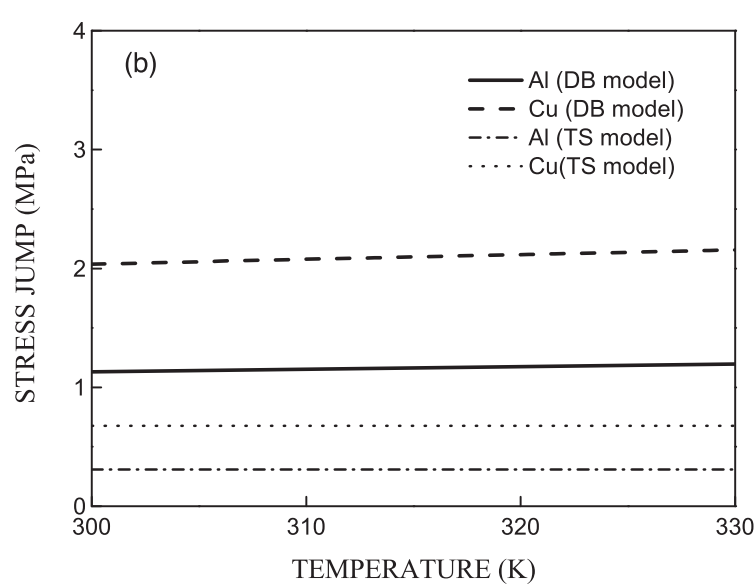

FIG. 13. (a) The yield stress jump $\delta \sigma_{\varphi}$ as a function of the dislocation density $\rho_{d}$ and plastic strain $\varepsilon$ according to the RIS models based on TSs (47) and DBs (48), compared to the experimental data for $\mathrm{Al}$ and $\mathrm{Cu}$ samples of a thickness $h=0.51 \mathrm{~mm}$. (b) Temperature dependence of $\delta \sigma_{\varphi}$ calculated for $\varepsilon=15 \%$. Material and irradiation parameters used in calculations are presented in Table II. 


\section{A. Time evolution of breathers}

Equation (6) is the simplest master equation for the DB distribution function, which implies that DBs are formed at a rate $K_{B}(E)$ and do not change for a time $\tau_{B}(E)$, after which they disappear. However, a breather can change its energy due to the interaction with phonons, electrons, other breathers, or stable lattice defects. This could be taken into account in the master equation of the following form:

$$
\frac{\partial f_{B}(E, t)}{\partial t}=K_{B}(E)-\frac{\partial}{\partial E}\left\lfloor f_{B}(E, t) \frac{d E}{d t}\right\rfloor-S_{B}(E),
$$

where $d E / d t$ is the adiabatically slow rate of breather energy change (e.g., due to phonons), and $S_{B}(E)$ is the DB sink in the energy space due to various collision events. This equation is similar to that used in the theory of nucleation and evolution of the new phase particles in a nonequilibrium environment (see, e.g., Ref. [22]). Evaluation of the master equation (50) is an outstanding problem in a more comprehensive theory.

\section{B. Moving breathers}

The interaction of moving DBs with defects is presently a subject of great interest and can be connected with a number of phenomena observed in crystals under irradiation, such as the radiation-induced migration of point defects in the crystal bulk $[23,24]$ or the radiation-induced formation of Schottky defects at extended crystal defects [25-27]. Thermally activated DBs considered in the present paper may move randomly from site to site, which would increase a probability of coupling between DBs and defects. This could be taken into account in the master equation (50).

Another type of mobile localized excitations (called quodons) are created in atomic collisions under irradiation with swift particles. As the incident recoil energy is dispersed, on-site potentials and long-range cooperative interactions between atoms can result in the creation of quodons, which are high-energy mobile longitudinal optical mode DBs that can propagate great distances in atomic-chain directions [25]. The interaction of quodons with extended lattice defects was suggested to result in the radiation-induced recovery processes such as the void shrinkage [26] and self-organization of the void lattices [27]. However, the underlying mechanism, i.e., the quodon-induced vacancy emission from voids, needs further investigations that should take into account the present results. This will be done elsewhere.

\section{SUMMARY}

(1) Reaction rate theory in solids has been modified taking into account intrinsic localized modes or discrete breathers that can appear in crystals with sufficient anharmonicity, resulting in violation of Arrhenius' law.

(2) The reaction rate averaged over large macroscopic volumes and times including many DBs can be increased by many orders of magnitude depending on the DB statistics.

(3) The breather statistics in thermal equilibrium and under irradiation with swift particles has been considered, and the corresponding reaction rate amplification factors have been derived.

(4) The reaction rate amplification factor increases strongly with increasing DB lifetime and the maximum DB energy that depends on the system.

(5) Radiation-induced formation of DBs changes mechanical properties of materials under reactor conditions as compared to the surveillance samples in out-reactor tests after an equivalent irradiation dose, which should be taken into account in forecasting the material service life.

(6) The present model shows that the effects due to the crystal anharmonicity are of both fundamental significance and of considerable technological importance in the fields of nuclear engineering and radiation effects.

\section{ACKNOWLEDGMENTS}

We thank M. Russell, C. Eilbeck, and S. Flach for interesting discussions. This work was supported in part (VD) by STCU-NASU Grant No. 4962. JFRA acknowledges financial support from MICINN project FIS2008-04848.
[1] A.J. Sievers and S. Takeno, Phys. Rev. Lett. 61, 970 (1988)

[2] R. S. MacKay and S. Aubry, Nonlinearity 7, 1623 (1994).

[3] J. F. R. Archilla, J. Cuevas, M. D. Alba, M. Naranjo, and J. M. Trillo, J. Phys. Chem. B 11024112 (2006).

[4] F. Piazza, S. Lepri, and R. Livi, Chaos 13, 637 (2003).

[5] S. Flach and A. V. Gorbach, Phys. Rep. 467, 1 (2008).

[6] P. Hanggi, P. Talkner, and M. Borkovec, Rev. Mod. Phys. 62, 251 (1990).

[7] L. Gammaitoni, P. Hanggi, P. Jung, and F. Marchsoni, Rev. Mod. Phys., 70, 223, (1998).

[8] M. V. Ivanchenko, O. I. Kanakov, V. D. Shalfeev, and S. Flach, Phys. D 198, 120 (2004).

[9] R. L. Fleischer, P. B. Price, and R. M. Walker, Nuclear Tracks in Solids (University of California, Berkeley, 1979).

[10] M. W. Finnis, P. Agnew, and A. J. E. Foreman, Phys. Rev. 44, 567 (1991)
[11] A. E. Volkov and V. A. Borodin, Nucl. Instrum. Methods Phys. Res. B 146, 137 (1998).

[12] I. M. Lifshits, M. I. Kaganov, and L. V. Tanatarov, Atom. Energy 6, 391 (1959) (in Russian).

[13] V. I. Dubinko, V. F. Klepikov, V. E. Noviokv, P. N. Ostapchuk, A. A. Soroka, L. V. Tanatarov, and I. V. Tanatarov, Problems of Atomic Science and Technology, 90(2), 48 (2007) (in Russian).

[14] V. I. Dubinko, S. A. Kotrechko, and V. F. Klepikov, Radiat. Eff. Defects Solids 164, 647 (2009).

[15] O. A. Troitskii and V. I. Likhtman, Dokl. Akad. Nauk S.S.S.R. 148, 332 (1963) (in Russian).

[16] O. A. Troitskii and V. I. Spitsyn, Metallofizika, 51, 18 (1974) (in Russian).

[17] V. I. Dubinko, Technical report 3 of STCU project 4368 (2009). 
[18] V. I. Dubinko, A. N. Dovbnya, V. A. Kushnir, V. V. Mitrochenko, I. V. Khodak, V. P. Lebedev, V. S. Krylovsky, and V. F. Klepikov, Problems of Atomic Science and Technology, 54(3), 140 (2010) (in Russian).

[19] V. I. Dubinko, V. I. Karas, V. F. Klepikov, P. N. Ostapchuk, and I. F. Potapenko, Problems of Atomic Science and Technology, 94(4), 158 (2009) (in Russian).

[20] N. I. Isaev, V. D. Natsik, and V. F. Fomenko, Fizika Nizkikh Temperatur 25, 987 (1999) (in Russian).

[21] R. W. Cahn and P. Haasen (editors), Physical Metallurgy (North-Holland Physics Publishing, Amsterdam, 1983).
[22] V. I. Dubinko, A. A. Turkin, D. I. Vainshtein, and H. W. den Hartog, J. Nucl. Mater. 304, 117 (2002).

[23] J. Cuevas, C. Katerji, J. F. R. Archilla, J. C. Eilbeck, and F. M. Russell, Phys. Lett. 315, 364 (2003).

[24] G. Abrasonis, W. Moller, and X. X. Ma, Phys. Rev. Lett. 96, 065901 (2006).

[25] F. M. Russell and J. C. Eilbeck, Europhys. Lett. 78, 10004 (2007).

[26] V. I. Dubinko, A. G. Guglya, E. Melnichenko, and R. Vasilenko, J. Nucl. Mater. 385, 228 (2009).

[27] V. I. Dubinko, Nucl. Instrum. Methods Phys. Res. B 267, 2976 (2009). 